\title{
Antibacterial activity of Amikacin-immobilized detonation nanodiamond
}

\author{
A. S. Solomatin ${ }^{1,2}$, R. Yu. Yakovlev ${ }^{1,2,3}$, O. V. Efremenkova ${ }^{4}$, \\ I. G. Sumarukova ${ }^{4}$, I. I. Kulakova ${ }^{2}$, G. V. Lisichkin ${ }^{2}$ \\ ${ }^{1}$ Ryazan State Medical University, Ryazan, Russia \\ ${ }^{2}$ Lomonosov Moscow State University, Moscow, Russia \\ ${ }^{3}$ Vernadsky Institute of Geochemistry and Analytical Chemistry of Russian Academ of Sciences, \\ Moscow, Russia \\ ${ }^{4}$ Gause Institute of New Antibiotics, Moscow, Russia \\ solo-msu@yandex.ru, yarules@yandex.ru, ovefr@yandex.ru, \\ noks59@yandex.ru, inna-kulakova@yandex.ru, lisich@petrol.chem.msu.ru
}

PACS 68.35.-p, 7.85.Tu

DOI 10.17586/2220-8054-2017-8-4-531-534

Detonation nanodiamonds (NDs) with chlorinated (ND-Cl) and carboxylated (ND-COOH) surfaces were obtained. The broad-spectrum antibiotic Amikacin (Amik) was covalently grafted to the chlorinated surface (ND-Amik) and immobilized by adsorption to carboxylated surface (Amik/ND-COOH). Biological testing in vitro showed the presence of antibacterial activity of the obtained samples against Staphylococcus aureus FDA P209 and Escherichia coli ATCC 25922, close to activity of free amikacin. It was revealed that to maintain antibacterial activity of the samples after their preliminary treatment, important factors such as the use of antioxidants (hydrosulfite and sodium citrate) and lyophilization were necessary.

Keywords: detonation nanodiamond (ND), amikacin (Amik), lyophilization, antioxidants, antibacterial activity.

Received: 27 May 2017

Revised: 3 July 2017

\section{Introduction}

The development of effective systems of drugs delivery is one of the rapidly developing areas of modern chemistry, pharmacology and medicine [1-3]. Recent research has shown that detonation nanodiamond (ND) is a promising nanocarrier to create delivery systems. It is non-toxic, biocompatible and able to overcome biological barriers, penetrating into the cells [4]. The high concentration of functional groups on its surface allows to use of a wide range of reactions to modify the nanodiamond [5,6] and immobilize many different biologically active substances (BAS) on it. The adsorption [7] and the covalent grafting [8] are methods commonly used for the immobilization of BAS on ND. A number of studies have shown that the activities of BAS are preserved and their toxicities may be attenuated with immobilization on ND $[9,10]$. One of the most effective antibacterial aminoglycosides used for the treatment of various infectious diseases, including tuberculosis, is amikacin. This compound is a broad-spectrum antibiotic and antituberculosis drugs II series [11,12]. Both ototoxicity and nephrotoxicity are known characteristics of amikacin, as with other aminoglycoside antibiotics [13], however, to a lesser degree [14].

The problem of obtaining of a highly efficient ND-based delivery system for amikacin to reduce its dosage while enhancing the efficiency its action, especially with respect to phthisiopulmonology [11], is highly relevant and challenging. Therefore the goal of this work was to study the antibacterial activity of Amikacin-immobilized detonation nanodiamonds.

\section{Experimental}

\subsection{Materials and methods}

Amikacin (Amik) is used in the active pharmaceutical compound "Amikacin sulphate" (JSC "Sintez", Kurgan, Russia), which is a white lyophilized powder for the preparation of injection solutions. Nanodiamond of detonation synthesis (trademark UDA-TAN) were acquired in SKTB “Tekhnolog” (St. Petersburg, Russia). In accordance with the specification, ND contained $0.7 \mathrm{wt} . \%$ non-combustible impurities. In order to reduce the impurities content ND was sequentially treated with aq. $0.1 \mathrm{M} \mathrm{NaOH}$ solution and concentrated $\mathrm{HCl}$, thoroughly rinsed with water and dried [15].

Reagents with purity “cm. p" and "v. p.": $\mathrm{H}_{2} \mathrm{SO}_{4}, \mathrm{HNO}_{3}, \mathrm{HCl}, \mathrm{NaOH}, \mathrm{NaHSO}_{3}, \mathrm{KMnO}_{4}, \mathrm{CCl}_{4}, \mathrm{EtOH}, \mathrm{N}, \mathrm{N}-$ diisopropylethylamine, boric acid, 2,4,6-trinitrobenzenesulfonic acid (TNBS), and sodium citrate were purchased 
in Sigma. Hydrogen gas of purity $99.99 \%$; liquid nitrogen; nutrient medium No. 2 gauze for deep cultivation (composition, wt.\%: glucose -1.0 , peptone -0.5 , tripton -0.3 , sodium chloride -0.5 , water -97.7 ) and with the addition of $3 \mathrm{wt} . \%$ agar, exhibiting planting.

The structural characteristics and the size of the primary ND particles were examined by Transmission Electron Microscope JEM-2100 F ("JEOL", Japan). The specific surface area and porosity of the NDs were measured by BET using Gimini 2390 (V1.02 t) (Micromeritics, USA). The ND powder was pre-heated at $120^{\circ} \mathrm{C}$ and $10^{-3} \mathrm{~mm} \mathrm{Hg}$. The accuracy was $5 \mathrm{~m}^{2} / \mathrm{g}$. The analysis of surface functional groups was verified by Fourier Transform Infrared Spectroscopy (FTIR) using Nicolet IR200 (ThermoScientific, USA) with a spectra resolution $2 \mathrm{~cm}^{-1}$. ND $\left(0.8 \mathrm{mg}\right.$ ) was mixed with $140 \mathrm{mg}$ of $\mathrm{KBr}$ (previously dried at $100^{\circ} \mathrm{C}$ and $1 \mathrm{~mm} \mathrm{HG}$ ), and then finely ground by the grinding. The chemical composition of NDs surface was analyzed by X-ray photoelectron spectroscopy (XPS) using LAS-3000 (Riber, France) with $\mathrm{Al} \mathrm{K}_{\alpha}=1486.6 \mathrm{eV}$ X-rays at the voltage on the tube $12 \mathrm{kV}$ and emission current $20 \mathrm{~mA}$. Photoelectron peaks were calibrated using the line of $\mathrm{C} 1 \mathrm{~s}$-electron of carbon with a binding energy of $285 \mathrm{eV}$.

\subsection{Techniques}

The functionalization of ND (chlorination and carboxylation) and the subsequent covalent and adsorption immobilization of amikacin on those functionalized NDs, affording ND-Amik and Amik/ND-COOH, respectively, were performed according to previously described techniques [16]. The ND-Amik conjugate was obtained by covalent grafting of amikacin to the ND-Cl.

2.2.1. Hydrogenation and Chlorination of ND surface. The nanodiamond with hydrogen-containing surface groups (ND-H) was prepared by thermal gaseous hydrogenation, as described previously [16]. A quartz reactor with a sample of purified ND was placed in a tube furnace, heated to $800^{\circ} \mathrm{C}$ and held for $5 \mathrm{~h}$ in a stream of $\mathrm{H}_{2}$ (at 2-3 liter/h flow rate). The nanodiamond with chlorinated surface (ND-Cl) was prepared from ND-H. Chlorine was generated by reaction of $\mathrm{KMnO}_{4}$ with concentrated $\mathrm{HCl}$; the liberated gas was bubbled through carbon tetrachloride to produce a saturated solution (at $5.6 \mathrm{wt} . \%)$. ND-H $\left(500 \mathrm{mg}\right.$ ) was added to $40 \mathrm{ml}$ of $\mathrm{Cl}_{2}$ solution in $\mathrm{CCl}_{4}$. The resulting mixture was irradiated with an incandescent lamp $(500 \mathrm{~W})$ at constant stirring for $24 \mathrm{~h}$. Then the $\mathrm{ND}-\mathrm{Cl}$ was separated from the solution, rinsed with $\mathrm{CCl}_{4}$, dried using a rotary evaporator and degassed under vacuum $(<1$ bar $)$ for $2 \mathrm{~h}$.

2.2.2. Liquid-phase oxidation of $N D$ surface. The liquid-phase oxidation was carried out in a glass flask equipped with a reflux condenser. ND powder $(1 \mathrm{~g})$ was added to $75 \mathrm{ml}$ of $\mathrm{HNO}_{3}$ and $\mathrm{H}_{2} \mathrm{SO}_{4}(1: 3 \mathrm{v} / \mathrm{v})$. The resulting mixture was stirred for 24 hours at $120^{\circ} \mathrm{C}$. Carboxylated ND (ND-COOH) was separated by centrifugation for $10 \mathrm{~min}$ at $1000 \mathrm{~g}$. The precipitate was treated with $0.1 \mathrm{M} \mathrm{NaOH}$ for 2 hours at $90^{\circ} \mathrm{C}$, then with $0.1 \mathrm{M} \mathrm{HCl}$ for 2 hours at $90{ }^{\circ} \mathrm{C}$. Such resulting precipitate was separated by centrifugation and repeatedly washed with water. Then, ND-COOH was dried at $60{ }^{\circ} \mathrm{C}$ using a rotary evaporator.

\subsection{Preparation of ND hydrosols}

Hydrosols of ND-COOH and the other samples were prepared according to the procedure described in [17]: $600 \mathrm{mg}$ of nanodiamond powder was placed in a plastic centrifugation tube, and then suspended in $40 \mathrm{ml}$ of water by 1-min sonication. The suspension was then centrifuged at $6000 \mathrm{~g}$ for $5 \mathrm{~min}$. This cycle was repeated five times. The amount of ND in supernatant was determined by drying $0.5 \mathrm{ml}$ of hydrosol on the watch glass that was previously adjusted to a constant weight. The watch glass with a hydrosol was placed on a metal grid fixed 2-3 cm above electric hot plates heated at $200^{\circ} \mathrm{C}$. ND was dried to a constant weight.

The content of the immobilized amikacin on ND samples was determined by UV spectrophotometry using the preliminary derivatization with TNBS. For this purpose, the test sample hydrosols were diluted to ND concentration of $80 \mu \mathrm{g} / \mathrm{ml}$. The pre-prepared borate-sodium buffer $(\mathrm{pH} 9.5)$ and TNBS $(30 \mathrm{mM})$ were added to the hydrosol to concentrations in the resulting mixture, $0.1 \mathrm{M}$ and $300 \mu \mathrm{M}$, respectively. The mixture was stirred on Vortex at $40{ }^{\circ} \mathrm{C}$ for $1 \mathrm{~h}$. The optical density of the mixture was determined at a wavelength of $405 \mathrm{~nm}$ and the absorption contribution of the ND was subtracted. The content of antibiotic in the investigated hydrosols samples was determined using calibration curves, previously constructed for solutions of free amikacin.

The Amik/ND-COOH hydrosol was divided into two parts. The first part was dried on a rotary evaporator and, prior to testing the antibacterial activity, redispersed in distilled water by ultrasonication (Sample 1a). A solution of antioxidants (sodium hydrosulfite and sodium citrate, $\mathrm{pH} 4.5$ ) was added to the second aliquot to concentrations of 0.13 and $0.60 \mathrm{wt} . \%$ respectively. The mixture was sprayed into liquid nitrogen using a hand sprayer and lyophilized. The lyophilisate was redispersed in distilled water and sterilized using UV irradiation (Sample 1b). 
The ND-Amik hydrosol was divided into three parts. The Sample $2 b$ and the Sample 2c were obtained according to the techniques described for Sample 1b, except that Sample 2c was not UV-irradiated. The third part of the ND-Amik hydrosol was treated similarly to the preparation of Sample $2 b$, but without the addition of antioxidants (Sample 2d). As a reference sample (Control), free amikacin treated similarly to Sample $1 \mathrm{~b}$ was used.

\subsection{Antibacterial activity of Amikacin-immobilized nanodiamonds}

The antibacterial activity of the prepared samples was studied by a serial dilution method in medium (deep culture in vitro) with respect to Staphylococcus aureus FDA P209 and Escherichia coli ATCC 25922. The liquid N2 Gause medium was inoculated with test strains at a calculated value of $10^{7}$ bacteria/ml (calculated according to the McFarland turbidity standard). Survival of bacteria was assessed by spots on an agar-agar N2 Gause medium. Preliminarily, the minimum inhibitory concentration (MIC) of the Control was determined in the concentration range $2-16 \mu \mathrm{g} / \mathrm{ml}$. Then, the same mixture of all investigated samples with the same concentrations of Amikacin were prepared and examined. Conclusions regarding the absence of antibacterial activity were made based on the presence of bacterial colonies on the surfaces of agar-agar media.

\section{Results and discussion}

Main characteristics of the test samples are shown in Table 1.

TABLE 1. Test samples of ND with immobilized amikacin

\begin{tabular}{|c|c|c|c|c|}
\hline Sample & $\begin{array}{c}\text { Method of immobilization } \\
\text { of Amik on ND }\end{array}$ & $\begin{array}{c}\text { Presence } \\
\text { of antioxidants }\end{array}$ & Drying method & $\begin{array}{c}\text { Amik } \\
\text { conmob } \\
\text { content, wt.\% }\end{array}$ \\
\hline 1a & Adsorption & - & ordinary & 3.1 \\
\hline 1b & - -" & + & lyophilization & 3.1 \\
\hline 2b & Covalent grafting & + & - -"- & 4.3 \\
\hline 2c & -"- & + & - -"- & 4.3 \\
\hline 2d & -"- & - & - -"- & 4.3 \\
\hline
\end{tabular}

The amount of amikacin immobilized on ND was small, so it is practically below the threshold limit for detection in IR absorption spectra. Therefore, UV-spectroscopy was used as the main method for amikacin determination. Because amikacin has no absorption maxima absorption in the UV range, it was derivatized with TNBS, which forms salts with the amino groups of the antibiotic and allows detection of absorption at $405 \mathrm{~nm}$.

The purpose of antioxidants addition was to verify the assumption of their positive effect on the stability of amikacin. So the mass ratio amikacin/ $\mathrm{NaHSO}_{3}$ and amikacin/citrate was equal to 40 and 8 , respectively (as in pharmacy amikacin for injection).

The results of the antibacterial activity study for the test samples are summarized in Table 2 . From a comparison of the presented data, it follows that the MIC of the Control in relation to S. Aureus FDA P209 and E. coli ATCC 25922 were 8 and $16 \mu \mathrm{g} / \mathrm{ml}$, respectively. Sample 1a showed no antibacterial activity in the investigated concentration range for any bacterial strain.

TABLE 2. Influence of the test samples content in a culture medium (Amik concentration) on the growth of $S$. aureus FDA P209 and E. coli ATCC 25922: "-" no growth, "+" growth. Bacteria incubation with the samples was carried out at $37^{\circ} \mathrm{C}$ for $24 \mathrm{~h}$

\begin{tabular}{|c|c|c|c|c|c|c|c|c|}
\hline \multirow{3}{*}{ Sample } & \multicolumn{4}{|c|}{ St. aureus FDA P209 } & \multicolumn{5}{c|}{ E. coli ATCC 25922 } \\
\cline { 2 - 10 } & \multicolumn{4}{|c|}{ C(Amik), $\mu$ g/ml } & \multicolumn{4}{c|}{ C(Amik), $\mu$ g/ml } \\
\cline { 2 - 10 } & $\mathbf{2}$ & $\mathbf{4}$ & $\mathbf{8}$ & $\mathbf{1 6}$ & $\mathbf{2}$ & $\mathbf{4}$ & $\mathbf{8}$ & $\mathbf{1 6}$ \\
\hline $1 \mathrm{a}$ & + & + & + & + & + & + & + & + \\
\hline $1 \mathrm{~b}$ & + & + & + & - & + & + & + & + \\
\hline $2 \mathrm{~b}$ & + & + & + & - & + & + & + & - \\
\hline $2 \mathrm{c}$ & + & + & + & - & + & + & + & - \\
\hline $2 \mathrm{~d}$ & + & + & + & + & + & + & + & + \\
\hline Control & + & + & - & - & + & + & + & - \\
\hline
\end{tabular}


The antioxidant-containing and lyophilized Amik/ND-COOH (Sample 1b) showed a suppressive effect with respect to $S$. aureus at $16 \mu \mathrm{g} / \mathrm{ml}$, whereas at the same concentration, it had no noticeable effect with respect to E. coli. ND-Amik with antioxidants (Sample 2b) showed antibacterial activity against both strains, and its effect on E. coli is comparable to Control. Similar results for Samples $2 \mathrm{~b}$ and $2 \mathrm{c}$ show that irradiation with UV-light (sterilization) has no effect of the biological experiment result, since, on one hand, it does not affect the activity of the sample, and, on the other, the growth of extraneous organisms, which affirms the sterility of the sample and the unnecessariness for additional sterilization. The Sample 2d (lyophilized ND-Amik, but without the addition of antioxidants) showed no antibacterial activity in the studied concentrations range. Thus, lyophilization and the use of antioxidants are important factors in preserving the activity of amikacin immobilized on ND in the delivery system.

\section{Conclusions}

It has been established that samples of amikacin covalently immobilized on nanodiamonds possess the greatest antibacterial activity. The activity of such samples is close to the activity of free amikacin, which is quite acceptable for delivery systems. It is shown that the biological activity of ND conjugates with amikacin is influenced not only by the method of immobilization, but also by other factors, including, primarily, the presence of antioxidants and lyophilic drying. In turn, the use of these additional factors makes it possible to more fully reveal the potential of the BAS delivery systems based not only on ND, but also on other nanoparticles.

\section{Acknowledgments}

This study has been performed with support from the grant of Russian Science Foundation (project 16-0801156). The equipment used in this study was purchased from the funds of Moscow State University Development Program.

\section{References}

[1] Nanotherapeutics: Drug Delivery Concepts in Nanoscience. Edited by Lamprecht A. CRC Press, Taylor and Francis Group, Boca Raton, 2008, 292 pp.

[2] Almjasheva O.V., Garabadzhiu A.V., Kozina Yu.V., Litvinchuk L.F., Dobritsa V.P. Biological effect of zirconium dioxidebased nanoparticles. Nanosystems: physics, chemistry, mathematics, 2017, 8(3), P. 391-396.

[3] Popova N.R., Popov A.L., Shcherbakov A.B., Ivanov V.K. Layer-by-layer capsules as smart delivery systems of $\mathrm{CeO}_{2}$ nanoparticle based theranostic agents. Nanosystems: physics, chemistry, mathematics, 2017, 8(2), P. 282-289.

[4] Zhang X., Hu W., Li J., Tao L., Wei Y. A comparative study of cellular uptake and cytotoxicity of multi-walled carbon nanotubes, graphene oxide, and nanodiamond. Toxicol. Res., 2012, 1, P. 62-68.

[5] Krueger A. The structure and reactivity of nanoscale diamond. J. Mater. Chem., 2008, 18, P. 1485-1492.

[6] Shenderova O.A., McGuire G.E. Science and engineering of nanodiamond particle surfaces for biological applications (Review). Biointerphases, 2015, 10(3), P. 030802.

[7] Shugalei I.V., Voznyakovskii A.P., Garabadzhiu A.V., Tselinskii I.V., Sudarikov A.M., Ilyushin M.A. Biological activity of detonation nanodiamond and prospects in its medical and biological applications. Russ. J. Gen. Chem., 2013, 83(5), P. 851-883.

[8] Liu K.K., Zheng W.W., Wang C.C., Chiu Y.C., Cheng C.L., Lo Y.S., Chen C., Chao J.I. Covalent linkage of nanodiamond-paclitaxel for drug delivery and cancer therapy. Nanotechnology, 2010, 21, P. 1-14.

[9] Chen M., Pierstorff E.D., Lam R., Li S.Y., Huang H., Osawa E., Ho D. Nanodiamond-mediated delivery of water-insoluble therapeutics. ACS Nano, 2009, 3(7), P. 2016-2022.

[10] Chow E.K., Zhang X.Q., Chen M., Lam R., Robinson E., Huang H., Schaffer D., Osawa E., Goga A., Ho D. Nanodiamond therapeutic delivery agents mediated enhanced chemoresistant tumor treatment. Sci. Tran. Med., 2011, 3(73), P. 73 ra21.

[11] Pham D.D., Fattal E., Tsapis N. Pulmonary drug delivery systems for tuberculosis treatment. Int. J. Pharm., 2015, 478, P. 517-529.

[12] Xie J., Talaska A.E., Schacht J. New developments in aminoglycoside therapy and ototoxicity. Hear Res., 2011, 281, P. $28-37$.

[13] Brummetti R.E., Fox K.E. Aminoglycoside-induced hearing loss in humans. Antimicrob Agents Chemother., 1989, 33(6), P. 797-800.

[14] Hottendorf G.H., Gordon L.L. Comparative low-dose nephrotoxicities of gentamicin, tobramycin, and amikacin. Antimicrob. Agents Chemother, 1980, 18(1), P. 176-181.

[15] Method for selective final purification of nanodiamond, Patent. 2506095 Russia: MPK A61 K 47/04, C01 B 31/06, B82 B 3/00, B82 Y 5/00, Jakovlev R.J., Solomatin A.S., Kulakova I.I., Lisichkin G.V., Korolev K.M., Leonidov N.B., N 2012157038/15, 12 pp.

[16] Yakovlev R.Y., Solomatin A.S., Leonidov N.B., Kulakova I.I., Lisichkin G.V. Detonation diamond - a perspective carrier for drug delivery systems. Rus. J. Gen. Chem., 2014, 84(2), P. 379-390.

[17] Yakovlev R.Y., Osipova A.S., Solomatin A.S., Kulakova I.I., Murav'eva G.P., Avramenko N.V., Leonidov N.B., Lisichkin G.V. An approach to unification of the physicochemical properties of commercial detonation nanodiamonds. Russ. J. Gen. Chem., 2015, 85(6), P. $1565-1574$. 